\title{
Magnetization Switching in Nanowires: Monte Carlo Study with Fast Fourier Transformation for Dipolar Fields
}

\author{
D. Hinzke and U. Nowak \\ Theoretische Tieftemperaturphysik, Gerhard-Mercator-Universität Duisburg, 47048 Duisburg/ Germany \\ e-mail:denise@thp.uni-duisburg.de,uli@thp.uni-duisburg.de
}

(February 9, 2000)

\begin{abstract}
For the investigations of thermally activated magnetization reversal in systems of classical magnetic moments numerical methods are desirable. We present numerical studies which base on time quantified Monte Carlo methods where the long-range dipole-dipole interaction is calculated with the aid of fast Fourier transformation. As an example, we study models for ferromagnetic nanowires comparing our numerical results for the characteristic time of the reversal process also with numerical data from Langevin dynamics simulations where the fast Fourier transformation method is well established. Depending on the system geometry different reversal mechanism occur like coherent rotation, nucleation, and curling.
\end{abstract}

Pacs: $75.10 . \mathrm{Hk}, 75.40 . \mathrm{Mg}, 75.40 . \mathrm{Gb}$

Keywords: Classical spin models, Numerical simulation studies, Thermal activation, Magnetization reversal, Nanostructures

\section{INTRODUCTION}

Small magnetic particles in the nanometer regime are interesting for fundamental research as well as for applications in magnetic devices. With decreasing size thermal activation becomes more and more important for the stability of nanoparticles and is hence investigated experimentally as well as theoretically. Wernsdorfer et al. studied magnetization reversal in nanowires [1] as well as in nanoparticles [2] experimentally. They found that for very small particles the magnetic moments rotate coherently as in the Stoner-Wohlfarth model [2] while for larger system sizes more complicated nucleation processes occur.

Numerical studies of the thermal activation in magnetic systems base either on Langevin dynamics [3 6] or on Monte Carlo methods. With the second method, mainly nucleation processes in Ising systems have been studied [7, 8], but also vector spin models have been used to investigate the switching behavior in systems with continuous degrees of freedom [9 13. In these numerical studies the dipole-dipole interaction is often neglected or at least approximated due to its large computational effort.

In this work, we will use Monte Carlo methods in order to investigate the thermally activated magnetization behavior of a systems of magnetic moments including the dipole-dipole interactions. Since the calculation of these long-range interaction is extremely time consuming when performed straightforward it is necessary to develop efficient numerical methods for this task. We will demonstrate that the implementation of fast Fourier transformation (FFT) methods - which are already established in the context of micromagnetic simulations based on the Landau-Lifshitz equation of motion [14,15 — is possible also in a Monte Carlo algorithm where a single-spin-flip method is used.

Our approach is applied to the important problems of thermally activated magnetization reversal in a model for nanowires. Depending on the system geometry, material parameters, and the magnetic field different reversal mechanisms occur. Very small wires reverse by a coherent rotation mode while for sufficiently long wires the reversal is dominated by nucleation 13, 16. With increasing system width an additional crossover sets in to a reversal by a curling-like mode. Our numerical results based on Monte Carlo simulations are supplemented by Langevin dynamics simulations for comparison.

\section{MODEL FOR A NANOWIRE}

We consider a classical Heisenberg Hamiltonian for localized spins on a lattice,

$$
\begin{aligned}
\mathcal{H} & =-J \sum_{\langle i j\rangle} \mathbf{S}_{i} \cdot \mathbf{S}_{j}-\mu_{s} \mathbf{B} \cdot \sum_{i} \mathbf{S}_{i} \\
& -w \sum_{i<j} \frac{3\left(\mathbf{S}_{i} \cdot \mathbf{e}_{i j}\right)\left(\mathbf{e}_{i j} \cdot \mathbf{S}_{j}\right)-\mathbf{S}_{i} \cdot \mathbf{S}_{j}}{r_{i j}^{3}},
\end{aligned}
$$

where the $\mathbf{S}_{i}=\boldsymbol{\mu}_{i} / \mu_{s}$ are three dimensional magnetic moments of unit length. The first sum represents the ferromagnetic exchange of the moments where $J$ is the coupling constant, the second sum is the coupling of the magnetic moments to an external magnetic field $B$, and the last sum represents the dipolar interaction. $w=\mu_{0} \mu_{s}^{2} /\left(4 \pi a^{3}\right)$ describes the strength of the dipoledipole interaction with $\mu_{0}=4 \pi \cdot 10^{-7} \mathrm{Vs}(\mathrm{Am})^{-1}$. We consider a cubic lattice with lattice constant $a$. The $\mathbf{e}_{i j}$ are unit vectors pointing from lattice site $i$ to $j$.

We model a finite, cylindric system with diameter $D$ and length $L$ (in number of lattice sites). All our simulations start with a configuration where all spins point up (into $z$ direction). The external magnetic field is antiparallel to the magnetic moments, hence favoring a reversal process. As in earlier publications 10 13] we measure the 
characteristic time of the reversal process, i. e. the time when the $z$ component of the magnetization of the system changes its sign averaged over either 100 (Langevin dynamics) or 1000 (Monte Carlo) simulation runs.

\section{NUMERICAL METHODS}

In the following we will mainly use a Monte Carlo method with a time step quantification as derived in [12] and later further discussed in [13]. The method is based on a single-spin-flip algorithm. The trial step of the algorithm is a random movement of the magnetic moment within a certain maximum angle. In order to achieve this efficiently we construct a random vector with constant probability distribution within a sphere of radius $R$ and add this vector to the initial moment. Subsequently the resulting vector is normalized. The trial step width $R$ is connected to a time scale $\Delta t$ according to the relation

$$
R^{2}=\frac{20 k_{\mathrm{B}} T \alpha \gamma}{\left(1+\alpha^{2}\right) \mu_{s}} \Delta t
$$

Using this relation one Monte Carlo step per spin (MCS) corresponds to the time interval $\Delta t$ of the corresponding Landau-Lifshitz-Gilbert equation in the high damping limit [12]. Throughout the paper we set $\Delta t=\frac{1}{40} \mu_{s} / \gamma$ and adjust $R$, except of the simulations for Fig. 11 where we have to fix $R$ and calculate $\Delta t$.

In order to compute the dipole-dipole interaction efficiently we use FFT methods [17] for the calculation of the long-range interactions [14, 15]. This method uses the discrete convolution theorem for the calculation of the dipolar field

$$
\mathbf{H}_{i}=\sum_{j \neq i} \frac{3 \mathbf{e}_{i j}\left(\mathbf{e}_{i j} \cdot \mathbf{S}_{j}\right)-\mathbf{S}_{j}}{r_{i j}^{3}} .
$$

This dipolar field can be rewritten in the form

$$
H_{i}^{\eta}=\sum_{\theta, j} W_{i j}^{\eta \theta} S_{j}^{\theta}
$$

where the Greek symbols $\eta, \theta$ denote the Cartesian components $x, y, z$ and the Latin symbols $i, j$ denote the lattice sites. $\left(W^{\eta \theta}\right)_{i j}$ are interaction matrices which only depend on the lattice. Since the lattice is translational invariant one can apply the discrete convolution theorem in order to calculate the Fourier transform of the dipolar field as

$$
H_{k}^{\eta}=\sum_{\theta} W_{k}^{\eta \theta} S_{k}^{\eta}
$$

Thus one first computes the interaction matrices $\left(W^{\eta \theta}\right)_{i j}$ and its Fourier transform $\left(W^{\eta \theta}\right)_{k}$. This task has to be performed only once before the simulation starts since the interaction matrices depend only on the lattice structure and, hence, remain constant during the simulation. For each given spin configuration the dipolar field can then be calculated by first performing the Fourier transform of the $\mathbf{S}_{i}$, second calculating the product above following Eq. 5, and third transforming the fields $\mathbf{H}_{k}$ back into real space resulting in the dipolar fields $\mathbf{H}_{i}$. Using FFT techniques the algorithm needs only of the order of $N \log N$ calculations 177 instead of $N^{2}$ calculations which one would need for the straightforward calculation of the double sum in Eq. 11.

In order to apply the convolution theorem the system has to be periodically and the range of the interaction has to be identical to the system size. Since we are interested in finite systems with open boundary conditions we use the zero padding method [17]. This method is based on a duplication of the system size where the original system is wrapped up with zero spins.

The FFT methods is well established as containing no further approximation in the context of micromagnetism [18]. There is however an additional problem concerning the implementation of this method to Monte Carlo algorithms since here the spins are not updated in parallel. Principally, in a Monte Carlo simulation the dipolar field has to be calculated after each single spin flip since the dipolar field at any site of the lattice depends on all other magnetic moments. Thus if one magnetic moment moves, the value of the whole dipolar field changes. Hence, in a usual Monte Carlo algorithm one would store the dipolar fields in an array which is updated after every accepted spin flip 19. Here, only the changes of the dipolar field due to the single spin update are calculated ( $N$ operations) resulting in a need of computation time which scales with $N^{2}$ per MCS.

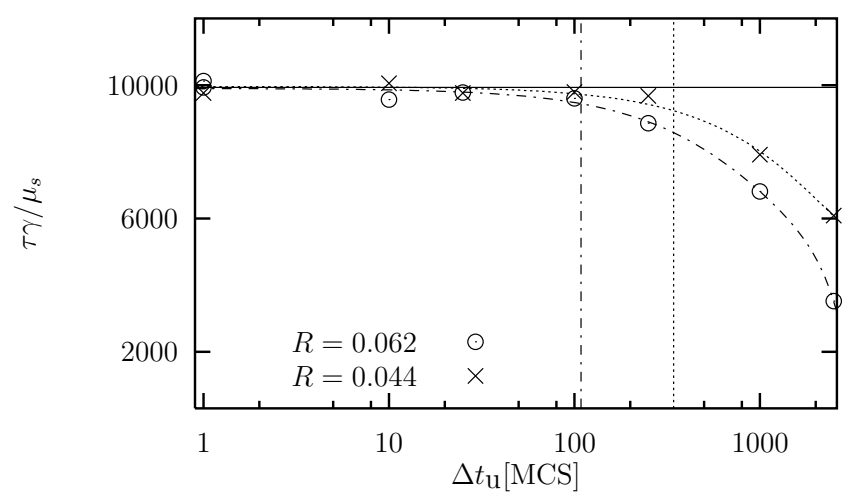

FIG. 1. Reduced characteristic time $\tau \gamma / \mu_{\mathrm{s}}$ vs. update interval $\Delta t_{\mathrm{u}}$ of the dipolar fields for different trial step widths $R$. The solid line represents the "correct" value when the dipolar field is calculated after every spin update. The vertical lines represent the number of MCS which one spin needs at least to reverse. Dashed lines connecting the points are guides to the eye. $L=8, \alpha=1, k_{\mathrm{B}} T=0.007 \mathrm{~J}, \mu_{s} B_{z}=0.15 \mathrm{~J}$, and $w=0.032 J$. 
Nevertheless, in the following we will show that alternatively one can also recalculate the whole dipolar field at once after a certain number of MCS taking advantage from the FFT method. This can be a good approximation as long as the changes of the system configuration during this update interval are small enough.

In order to investigate the validity of this idea systematically, we consider thermally activated magnetization reversal in a spin chain, in other words, in a cylinder with diameter $D=1$. First, we calculate the "correct" value of the reduced characteristic time following from a Monte Carlo simulation where the dipolar field is calculated after each accepted spin flip. This value is shown as solid line in Fig. 1. The data points represent the dependence of the reduced characteristic time on the length of the update interval $\Delta t_{u}$ after which the dipolar field is calculated. We used two different trial step widths $R$ for comparison.

As is demonstrated our method converges already for update intervals clearly longer than 1MCS, depending on the trial step width $R$. The dependence on $R$ can be understood as follows. The vertical lines represent the minimal number of MCS which one spin needs to reverse. This can be estimated from the mean step width of a magnetic moment 12] assuming that each trial step is accepted following a strong external field. The mean step width within our Monte Carlo procedure is $R / \sqrt{5}$ and thus the minimal number of MCS for a spin reversal is $\pi \sqrt{5} / R$. We conclude from this considerations that it is a good approximation to update the whole dipolar field after a certain interval of the Monte Carlo procedure which has to be smaller than the minimum number of MCS needed for a spin reversal. Note that it follows, that this method should never work for an Ising system.

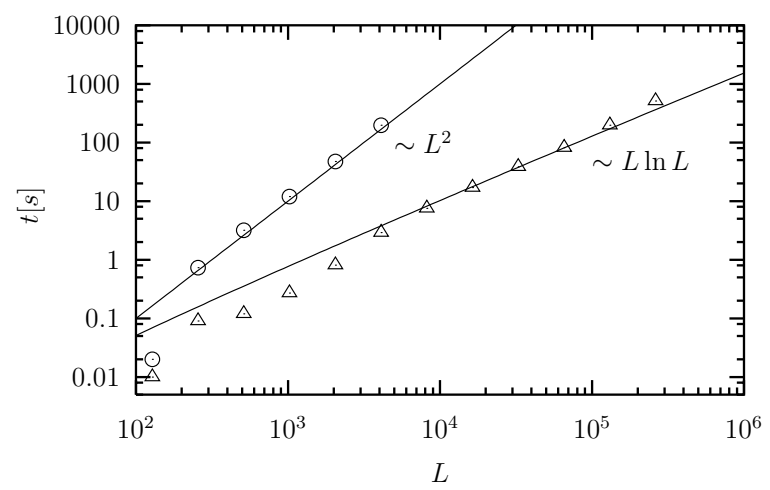

FIG. 2. Computation time $t$ vs. system size $L$ of a spin chain. The data are from Monte Carlo simulations where the dipolar field is calculated either directly as explained in the text (o) or with FFT methods $(\triangle)$.

Now we will turn to an investigation of the efficiency and capability characteristics of the method. A comparison of the computation time needed when the dipolar field is either calculated straightforward by updating the field after each accepted spin flip or with the FFT method after each MCS is shown in Fig. 2. Here, the computation time is the CPU time needed on an IBM RS6000/590 workstation for 100MCS. Using the FFT method the computation time is proportional to $L \ln L$ while for the usual method the computer time scales like $L^{2}$. For the chain of length $2^{18}=262144$ the gain of efficiency of the FFT method is roughly a factor 5000 (note that the FFT algorithm is most efficient for the system sizes which are products of small prime numbers) which is a rather impressing result.

As a test of the FFT method as well as the time quantification of the Monte Carlo algorithm we use for comparison also another numerical method namely Langevin dynamics simulations. Here we solve numerically the Landau-Lifshitz-Gilbert equation of motion with Langevin dynamics using the Heun method [5]. This equation has the form

$$
\frac{\left(1+\alpha^{2}\right) \mu_{s}}{\gamma} \frac{\partial \mathbf{S}_{i}}{\partial t}=-\mathbf{S}_{i} \times\left(\mathbf{H}_{i}(t)+\alpha\left(\mathbf{S}_{i} \times \mathbf{H}_{i}(t)\right)\right)
$$

with the internal field $\mathbf{H}_{i}(t)=\boldsymbol{\zeta}_{i}-\frac{\partial \mathcal{H}}{\partial \mathbf{S}_{i}}$, the gyromagnetic ratio $\gamma=1.76 \times 10^{11}(\mathrm{Ts})^{-1}$ and the dimensionless damping constant $\alpha$. The noise $\boldsymbol{\zeta}_{i}$ represents thermal fluctuations, with $\left\langle\boldsymbol{\zeta}_{i}(t)\right\rangle=0$ and $\left\langle\zeta_{i}^{\eta}(t) \zeta_{j}^{\theta}\left(t^{\prime}\right)\right\rangle=$ $2 \delta_{i j} \delta_{\eta \theta} \delta\left(t-t^{\prime}\right) \alpha k_{\mathrm{B}} T \mu_{s} / \gamma$ where $i, j$ denote once again lattice sites and $\eta, \theta$ Cartesian components. The implementation of the FFT method is straightforward for a Langevin dynamics simulation since here a parallel update of spin configurations is appropriate.

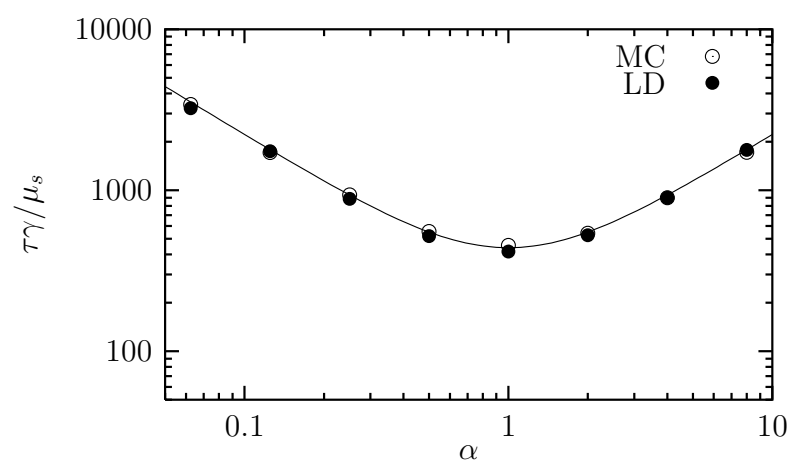

FIG. 3. Reduced characteristic time $\tau \gamma / \mu_{\mathrm{s}}$ vs. damping constant $\alpha$. The data are from Monte Carlo and Langevin dynamics simulations for a spin chain of length $L=64$. The line resembles the $\left(1+\alpha^{2}\right) / \alpha$ behavior. $\mu_{s} B_{z}=0.15 J, w=0.032 J$ and $k_{\mathrm{B}} T=0.025 J$.

In order to compare our different methods, in Fig. 3 the $\alpha$ dependence of the characteristic time for the reversal of a spin chain is shown. Monte Carlo data are compared with those from Langevin dynamics simulations. Interestingly, for the whole range of $\alpha$ values the Monte Carlo and Langevin dynamics data coincide. This is in contrast to earlier tests 12,13] where this agreement was achieved only in the high damping limit. Seemingly, 
there exist certain systems which show only a simple $\alpha$ dependence of the form $\tau \sim\left(1+\alpha^{2}\right) / \alpha$. This $\alpha$ dependence usually describes the high damping limit which is rendered by the time quantified Monte Carlo algorithm. In our model this $\alpha$ dependence is obviously valid in the whole range of damping constants which we considered, leading to the remarkable agreement. In general, this is not necessarily the case since for other models different low and high damping limits appear (see e. g. 20, 21]), depending on the systems properties. In the following we set $\alpha=1$.

\section{NUCLEATION AND CURLING}

As application of the methods described in the previous section we start with a simple chain of classical magnetic moments of length $L=128$ as extreme case of a cylindrical system with $D=1$. Thermally activated magnetization reversal in a spin chain was treated analytically by Braun [22, 23] who proposed a solitonantisoliton nucleation process as reversal mechanism. For a finite system with open boundary conditions the nucleation will originate at the sample ends leading to an energy barrier 24]

$$
\Delta E=2 \sqrt{2 d}(\tanh r-h r)
$$

with $h=\mu_{s} B_{z} /(2 d)$ and $r=\operatorname{arcosh} \sqrt{1 / h}$. Here, the dipole-dipole interaction is approximated by a uniaxial anisotropy of the form $-d \sum_{i}\left(S_{i}^{z}\right)^{2}$ with $w \approx d / \pi$ [24]. This estimate follows from a comparison of the stray field energy of an elongated ellipsoid [18] with the energy of a chain with uniaxial anisotropy. In [13] the occurrence of soliton-antisoliton nucleation was confirmed and Eq.7] was verified as well as the asymptotic form of the corresponding characteristic time

$$
\tau=\tau_{0} \exp \frac{\Delta E}{k_{\mathrm{B}} T}
$$

where $\tau_{0}$ is a prefactor depending on the system parameters, the applied magnetic field, and the damping constant. This prefactor was also derived asymptotically for a periodic chain for the case of nucleation [23] as well as for coherent rotation [25]. The latter is energetically favorable for low enough system size. However, for a finite chain the prefactor is still unknown.

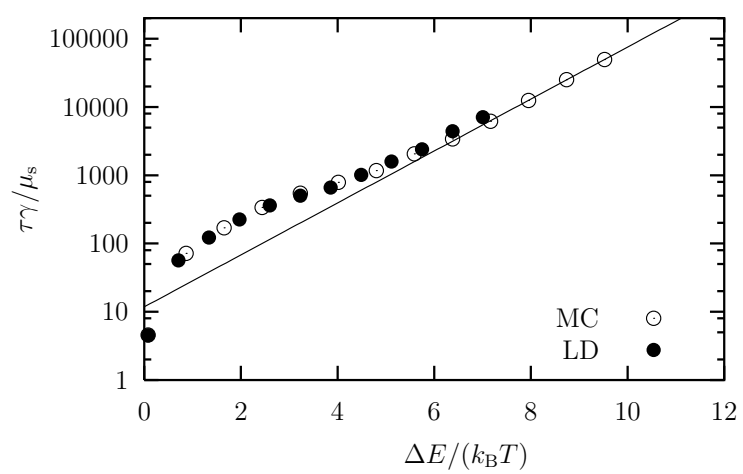

FIG. 4. Reduced characteristic time $\tau \gamma / \mu_{\mathrm{s}}$ vs. inverse temperature $\Delta E / k_{\mathrm{B}} T$ from Monte Carlo and Langevin dynamics simulations. The solid line is a fit to the low-temperature data in order to obtain the energy barrier numerically. $L=128$, $\mu_{s} B_{z}=0.15 J$, and $w=0.032 J$

Fig. 1 shows the temperature dependence of the reduced characteristic time for our spin chain where the dipole-dipole interaction is not approximated by a uniaxial anisotropy as discussed before [13 but taken into account using FFT methods. Data from Monte Carlo simulations are shown as well as from Langevin dynamics. Both methods yield identical results. The slope of the curve in the low temperature limit which represents the energy barrier is $12 \%$ lower than the predicted one. This deviation is probably due to the local approximation of the dipole-dipole interaction underlying Eq. 7.

We will now turn to the question whether such a nucleation mode will also be found in an extended system. Therefore we consider cylindrical systems of length 128 with varying diameter $D$. Fig. 5 shows snapshots of two simulated systems at the characteristic time. For $D=8$ (left side) two nuclei originate at the sample ends leading to two domain walls which pass the system. This is comparable to the nucleation process in a spin chain since all spins in a plane of the cylinder are more or less parallel (also shown in the figure) and can be describe as one effective magnetic moment leading to the above mentioned model of Braun 24.

The behavior changes for larger diameter above the socalled exchange length $l_{e x}$. For a reversal mode with inhomogeneous magnetization within the planes a domain wall has to be created. The loss of energy due to the existence of a $180^{\circ}$ domain wall on the length scale $l$ in a spin chain is

$$
\Delta E=J \sum_{i, j}\left(1-\cos \left(\theta_{i}-\theta_{j}\right)\right) \approx \frac{J \pi^{2}}{2 l}
$$

under the assumption that the change of the angle $\theta$ between the next nearest magnetic moments is constant (in a continuum description one can also prove that this resembles the minimum energy configuration by minimizing the Euler Lagrange equations). 


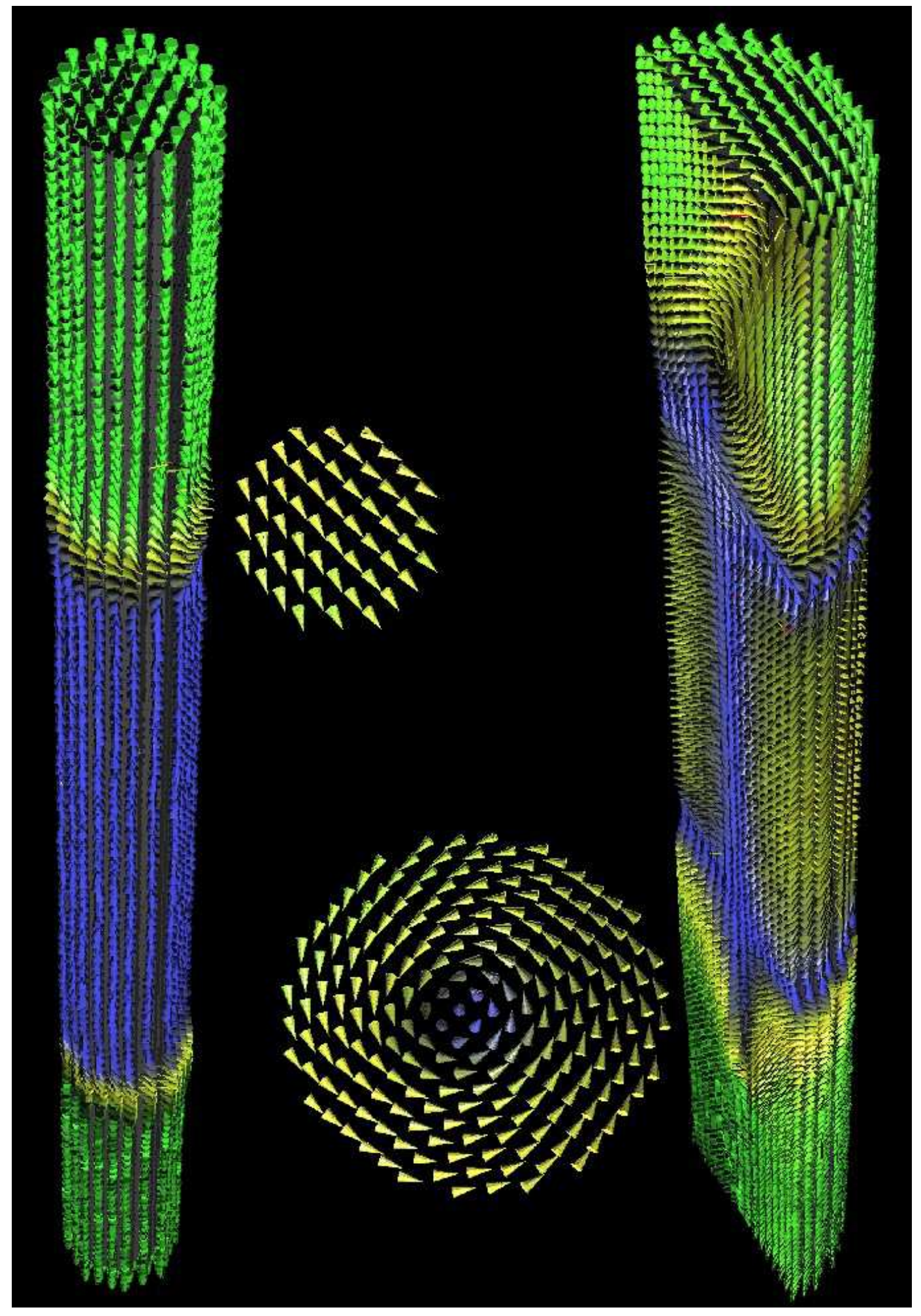

FIG. 5. Snapshots of simulated spin systems at the characteristic time for nucleation (left) and curling (right). The $z$ component of the magnetic moments is also color coded (green corresponds to spin down, blue to spin up). The clipping plane on the left side shows a layer in the upper domain wall that on the right side shows a layer with a magnetization vortex in the central curling region. $\mu_{s} B_{z}=0.15 J, w=0.032 J, L=128 . D=8$ (left) and 16 (right). 
The dipolar field energy of a chain of length $l$ is at most $3 w l \zeta(3)$ where we used Riemann's Zeta function

$$
\zeta(3)=\sum_{i}^{\infty} \frac{1}{i^{3}}
$$

(see also 19] for a corresponding calculation in two dimensions). A comparison of Eq. 9 and Eq. 10 yields the exchange length

$$
l_{\mathrm{ex}}=\pi \sqrt{\frac{J}{6 w \zeta(3)}} .
$$

Usually 18,24 the exchange length is calculated in the continuum limit where $3 \zeta(3) \approx 3.6$ is replaced by $\pi$. However, we prefer the slightly deviating expression above since its derivation is closer to the spin model which we discuss here. The exchange length for our material parameters is $l_{e x} \approx 7$, so that for systems with smaller diameter it cannot be energetically favorable for the system to build up inhomogeneous magnetization distributions within the planes while for wider systems another reversal process can occur namely curling [26, 27].

A snapshot during the reversal process in a wider particle $(D=16)$ is also shown in Fig. 5. The middle part of the cylinder is in the curling mode consisting of a magnetization vortex with a central axis which is still magnetized up. The reversal mode shown in this figure is still mixed up with an additional nucleation process which started first at the sample ends. Note, that in the clipping plane on the right hand side one can find the exchange length as typical length scale of the domain wall in the vortex.

\section{CONCLUSIONS}

We considered thermal activation in classical spin systems using Monte Carlo methods with a time quantified algorithm as well as Langevin dynamics simulations. We combined both techniques with FFT methods for the calculation of the dipolar field. Whereas this method is established in the context of micromagnetic equations of motion it is less obviously applicable to Monte Carlo methods with single spin flip dynamics. We show that it can be a good approximation for vector spins to update dipolar fields during a Monte Carlo procedure only after certain intervals the length of which depend on the trial step width of the algorithm. Since Monte Carlo methods need less computational effort as compared to Langevin dynamics simulations its combination with FFT methods strongly enhances the capabilities for the numerical investigation of thermal activation.

As application we consider a model for nanowires. We compare numerical results for the characteristic time of the magnetization reversal in a spin chain with the theoretical formula for the energy barrier of solitonantisoliton nucleation. We found small deviations probably due to the fact that Braun in his model approximated the dipole-dipole interaction by a uniaxial anisotropy [24. Varying the diameter in a cylindrical system we observe a crossover from nucleation to a curling-like mode.

\section{ACKNOWLEDGMENTS}

We thank H. B. Braun and K. D. Usadel for helpful discussions. The work was supported by the Deutsche Forschungsgemeinschaft, and by the EU within the framework of the COST action P3 working group 4.

[1] W. Wernsdorfer, B. Doudin, D. Mailly, K. Hasselbach, A. Benoit, J. Meier, J. P. Ansermet, and B. Barbara, Phys. Rev. Lett. 77, 1873 (1996).

[2] W. Wernsdorfer, E. B. Orozco, K. Hasselbach, A. Benoit, B. Barbara, N. Demoncy, A. Loiseau, H. Pascard, and D. Mailly, Phys. Rev. Lett. 78, 1791 (1997).

[3] A. Lyberatos and R. W. Chantrell, J. Appl. Phys 73, 6501 (1993).

[4] Y. Nakatani, Y. Uesaka, N. Hayashi, and H. Fukushima, J. Magn. Magn. Mat. 168, 347 (1997).

[5] J. L. García-Palacios and F. J. Lázaro, Phys. Rev. B 58, 14937 (1998).

[6] K. Zhang and D. R. Fredkin, J. Appl. Phys 85, 5208 (1999).

[7] P. A. Rikvold, M. A. Novotny, M. Kolesik, and H. L. Richards, in Dynamical properties of unconventional magnetic systems, edited by A. T. Skjeltrop and D. Sherrington (Kluwer, Dordrecht, 1998).

[8] M. Acharyya and D. Stauffer, Euro. Phys. J. B 5, 571 (1998).

[9] J. M. Gonzáles, R. Smirnov-Rueda, and J. González, J. Appl. Phys 81, 5573 (1997).

[10] D. Hinzke and U. Nowak, Phys. Rev. B 58, 265 (1998).

[11] U. Nowak and D. Hinzke, J. Appl. Phys 85, 4337 (1999).

[12] U. Nowak, R. W. Chantrell, and E. C. Kennedy, Phys. Rev. Lett. 84, 163 (2000).

[13] D. Hinzke and U. Nowak, Phys. Rev. B 61, (2000), in press.

[14] S. W. Yuan and H. N. Bertram, IEEE Trans. Mag. 28, 2031 (1992).

[15] D. V. Berkov, K. R. Ramstöck, and A. Hubert, Phys. stat. sol. (a) 137, 207 (1993).

[16] H. B. Braun, in Structure and Dynamics of Heterogenous Systems, edited by P. Entel and D. E. Wolf (World Scientific, Singapore, 2000).

[17] W. H. Press, B. P. Flannery, S. A. Teukolsky, and W. T. Vetterling, Numerical Recipes (Cambridge University Press, Cambridge, 1990). 
[18] A. Hubert and R. Schäfer, Magnetic Domains (SpringerVerlag, Berlin, 1998).

[19] A. Hucht, A. Moschel, and K. D. Usadel, J. Magn. Magn. Mat. 148, 32 (1995).

[20] W. T. Coffey, D. S. F. Crothers, J. L. Dorman, Y. P. Kalmykov, E. C. Kennedy, and W. Wernsdorfer, Phys. Rev. Lett. 80, 5655 (1998).

[21] W. T. Coffey, D. S. F. Crothers, J. L. Dorman, L. J.
Geoghegan, and E. C. Kennedy, Phys. Rev. B 58, 3249 (1998).

[22] H. B. Braun, Phys. Rev. Lett. 71, 3557 (1993).

[23] H. B. Braun, Phys. Rev. B 50, 16485 (1994).

[24] H. B. Braun, J. Appl. Phys 85, 6127 (1999).

[25] H. B. Braun, J. Appl. Phys 76, 6310 (1994)

[26] A. Aharoni, J. Appl. Phys 80, 3133 (1996).

[27] A. Aharoni, J. Appl. Phys 86, 1041 (1999). 